\title{
LA EDUCACIÓN NO FORMAL Desigualdades en el acceso a la formación de los trabajadores en España
}

\section{NON-FORMAL EDUCATION \\ Inequalities in the access to training for Spanish workers}

\author{
ISABEL GARCíA ESPEJo igarcia@uniovi.es \\ Universidad de Oviedo. España \\ Marta lbáÑez Pascual mibanez@uniovi.es \\ Universidad de Oviedo. España
}

\section{RESUMEN}

En un mundo con una fuerte innovación tecnológica y en el que la esperanza de vida y el tiempo de trabajo se alargan, la formación permanente deviene una necesidad productiva y social. A partir de la Encuesta sobre la Participación de la Población Adulta en las Actividades de Aprendizaje -EADA 2007- del Instituto Nacional de Estadística, en el presente artículo se utiliza una tipología de formación elaborada al efecto y poco empleada en nuestro entorno, que va más allá de las actividades de formación de las empresas al integrar las estrategias formativas de los trabajadores. Por medio de esta tipología y haciendo uso de una metodología basada en regresiones logísticas de eventos repetidos se han analizado los perfiles sociodemográficos de los trabajadores y los entornos organizativos que se asocian a cada tipo de formación. Los resultados muestran diferencias significativas en las variables estudiadas e indican que la formación laboral no reduce las desigualdades de partida de los trabajadores sino que tiende a mantenerlas e incluso a intensificarlas.

\section{Palabras Clave}

Aprendizaje permanente; Cualificaciones; EADA 2007.

\begin{abstract}
In a world with a strong technological innovation, increased life expectancy and more working time, lifelong learning becomes a productive and social need. From the Adult Education Survey -EADA is called AES in the EU - in the present manuscript, a formation typology that goes beyond the company formation activities due to integrate the workers training strategies, developed for that purpose and less used in our environment, has been employed. Through this typology and using a methodology based on logistic regressions of repeated measures, demographic employee profiles and organizational environments associated with each training type have been analyzed. The results show significant differences in the studied variables and indicate that training not reduces the starting inequalities, but tends to maintain and even intensify them.
\end{abstract}

\section{KEYWORDS}

Adult Education Survey (EU) AES; Lifelong Learning; Qualifications. 


\section{INTRODUCCIÓN}

La Unión Europea declara 1996 el Año Europeo de la Educación y de la Formación Permanente, seguramente el momento de mayoría de edad de una manera de mirar hacia la formación ya con cierta tradición entre las instituciones nacionales e internacionales, y con una presencia en la agenda pública cada día mayor. La Unesco popularizó este concepto a lo largo de las décadas de los 60 y 70 del pasado siglo, especialmente como una manera de ampliar la visión tradicional de la formación (educación formal) y dar carta de naturaleza a las formas de educación y formación de tipo no-formal o incluso informal. En el mismo sentido y en la misma época la OCDE incidía más sobre la idea de formación continua (recurrent education) implicando la necesidad de mejora de las cualificaciones de los trabajadores como vía de crecimiento económico. Por su parte, aunque los objetivos del año europeo son muy amplios ${ }^{1}$, se destaca ya la educación permanente como una pieza clave de la política comunitaria de empleo, teniendo en cuenta los fuertes cambios sociales y económicos que se están viviendo, la rápida transición hacia una sociedad basada en el conocimiento y, por último, el envejecimiento de la población europea (European Society of Association Education 2005). En suma, en el marco del actual Espacio Europeo de Educación Superior (EEES) es difícil no compartir la idea de la Comisión Europea de que la formación permanente se ha de constituir en un instrumento clave de competitividad. En este sentido, se espera que los diferentes agentes superen planteamientos reduccionistas, de manera que se convierta en un fenómeno general (Arís y Comas 2011)

La importancia de la formación permanente se refleja también en el monto de recursos que concentra, o en el número de personas que participan en ella. Por ejemplo, en el año 2007 tres de cada 10 personas de entre 25 y 64 años habían realizado alguna actividad formativa (Instituto Nacional de Estadística 2008a). Incluso, según datos del Ministerio de Educación, España se sitúa en niveles ligeramente superiores a la Unión Europea en participación de personas adultas en educación y formación. En concreto en 2010 , el $10,8 \%$ de los españoles de entre 25 y 64 años participaba en educación formal o no formal, mientras que el dato correspondiente a la Unión Europea 27 era del 9,1\% (Ministerio de Educación 2010).

Seguramente buena parte de la razón por la que la educación a lo largo de la vida es un fenómeno en aumento se deba también a su estrecha relación con el trabajo y el éxito profesional. Este efecto positivo de la formación sobre el empleo se ha convertido en un principio cada vez más aceptado en la sociedad y constituye incluso un tema recurrente

\footnotetext{
${ }^{1}$ En su declaración de intenciones la Unión Europea entiende que su contribución se caracteriza "por un enfoque amplio de «acompañamiento a lo largo de toda la vida», que no hace depender el aprendizaje de imperativos económicos y reconoce el derecho al enriquecimiento personal, la participación en el proceso democrático de toma de decisiones, el aprendizaje por propio interés y el envejecimiento activo." (Unión Europea 1996)
} 
en las investigaciones realizadas al efecto, las cuales se han centrado especialmente en su correlación positiva con las recompensas laborales (salarios, estabilidad) y la carrera profesional ascendente de los trabajadores, así como con el incremento de la productividad de las empresas, y, por ende, con el crecimiento y la capacidad económica de los países (European Centre for the Development of Vocational Training 2011; Huerta 2003; Barret y O'Connell 2001).

Pese a esta creencia, cada vez más extendida, en los beneficios de la formación, en España la proporción de empresas que invierten en estas actividades sigue siendo inferior al promedio de la UE - un $47 \%$ frente a un $60 \%$-, aunque se ha experimentado un importante crecimiento en los últimos años (European Centre for the Development of Vocational Training 2010). Como ejemplo, según los datos de la Fundación Tripartita para la Formación en el Empleo, en el año 2010, 380.548 empresas realizaron formación para sus trabajadores, lo que supone un incremento de casi el 30\% respecto a 2009 (Memoria 2010). Las características más significativas de esta formación en nuestro país son el protagonismo del sector de intermediación financiera, las diferencias por tamaño de las empresas a favor de las más grandes y la excesiva dependencia del sector empresarial de las subvenciones públicas (Mato 2010).

En este contexto, el objetivo principal del presente artículo es el de profundizar en el conocimiento del acceso diferencial de los trabajadores a la formación utilizando una base de datos con mucho potencial, la Encuesta sobre la Participación de la Población Adulta en las Actividades de Aprendizaje -EADA 2007. Precisamente el presente artículo es uno de los primeros trabajos que aborda el análisis estadístico y explotación de la citada fuente. Nuestra aproximación a la formación laboral de los trabajadores se llevará a cabo a través del concepto de educación no formal, (explicado en el siguiente apartado de este artículo), dividida a su vez entre formación financiada por la empresa, formación en el puesto de trabajo y formación realizada por iniciativa de los propios trabajadores, una tipología que nos permite ahondar en el fenómeno diferenciando las estrategias empresariales de las estrategias personales de aquellos trabajadores que se financian su propia formación (Escobar, Rivière y Glick 2010).

A partir de esta tipología, se analiza el perfil de los trabajadores y las características de las empresas o entornos organizativos en los que tienen lugar las decisiones de inversión formativa de empresas e individuos. En este sentido es interesante la separación de las distintas dimensiones que intervienen en el fenómeno a estudiar (personales, del puesto y de la empresa), pues según resalten unas características sobre otras podemos entender que la formación obedece más a la decisión personal, o bien, prevalecen factores estructurales que son los que determinan la probabilidad de recibir formación.

Asociado al primer objetivo de análisis se va a estudiar el efecto de la formación a la hora de igualar oportunidades; es decir, si la formación laboral reduce las desigualdades de partida de los individuos o por el contrario tiende a mantenerlas e incluso amplificarlas. Si, como ya hemos comentado, la formación proporciona rendimientos positivos a los trabajadores en cuanto a salario, posibilidades de promoción y desarrollo de trayec- 
torias laborales ascendentes, se trata de ver si todos estos tipos de formación benefician especialmente a aquellos que parten de mejores posiciones en cuanto a capital humano, aumentando así las desigualdades de partida en el mercado de trabajo.

Por último se abordan las dificultades u obstáculos que tuvieron algunos trabajadores para formarse, así como su perfil. Un problema muy poco estudiado por la literatura, seguramente porque hasta ahora no se contaba con información relevante, y cuyo conocimiento permitirá un mejor diseño de la formación para la población ocupada.

La estructura de este artículo es la siguiente: después de esta introducción, en el siguiente apartado se profundiza en el objeto de estudio, el aprendizaje no formal y su relación con el trabajo. La exposición del marco teórico y de las investigaciones que han abordado esta materia se lleva a cabo en el apartado tercero. En el apartado cuarto se desarrolla la metodología utilizada, para pasar seguidamente en el quinto apartado al análisis de los distintos tipos de formación. El estudio de los perfiles de los trabajadores que no se forman y sus razones son el objetivo del apartado sexto. El artículo finaliza con la presentación de las conclusiones más relevantes.

\section{SOBRE EL OBJETO DE ESTUdIO. EL APRENDIZAJE NO FORMAL Y SU RELACIÓN CON EL TRABAJO}

Como su propio nombre indica, el aprendizaje permanente (lifelong learning) se refiere a la búsqueda de conocimiento a lo largo de la vida; un esfuerzo voluntario y automotivado cuyas razones pueden ser tanto personales como profesionales. Esto significa que estamos hablando de un tipo de formación que no sólo refuerza el desarrollo personal, también puede tener un fuerte papel en la mejora del capital humano, aumentando la empleabilidad y la competitividad de los trabajadores en el mercado laboral. La UE entiende el aprendizaje a lo largo de la vida como: "toda actividad de aprendizaje emprendida a lo largo de la vida, con el objetivo de mejorar o ampliar conocimientos, habilidades y competencias, desde una perspectiva personal, cívica, social y/o relacionada con el empleo" (Instituto Nacional de Estadística 2008b:5); destacando en esta definición el carácter intencionado del aprendizaje ("búsqueda deliberada de conocimientos, habilidades, competencias o actitudes de valor permanente") y que dicho aprendizaje esté organizado, para lo que es imprescindible que tenga un agente proveedor y "un método de enseñanza a través del cual se organiza la comunicación", aunque también se incluye la posibilidad de que pueda ser autoorganizada (2008b:6).

Por su parte, es común clasificar la formación en cuatro grandes tipos (y así lo hace la Unión Europea y las encuestas auspiciadas por Eurostat): educación formal, educación no formal, aprendizaje informal y aprendizaje aleatorio. Los dos últimos tipos no cumplirían las condiciones del concepto "aprendizaje a lo largo de la vida", pues el aprendizaje aleatorio es el que se obtiene sin la intención expresa o la voluntad de conseguirlo, por ejemplo por la mera exposición a los medios de comunicación. Mientras 
que el aprendizaje informal se define como intencionado pero es menos organizado y menos estructurado, permite adquirir conocimientos a través de la práctica diaria y la relación con el medio, como, por ejemplo, el aprendizaje de labores domésticas en el entorno familiar.

La educación formal constituye un caso sui géneris y ciertamente minoritario en el fenómeno de la formación permanente por la dedicación a la que obliga. La educación formal recoge los distintos sistemas educativos cronológicamente graduados, estructurados y jerarquizados que se concretan en un currículo oficial. Supone una trayectoria ascendente de formación a tiempo completo (educación primaria, secundaria y superior) con una cronología obligatoria de los 6 a los 16 años para primaria y secundaria de primer ciclo, y que en los niveles de secundaria de segundo ciclo y universitarios se concentra en las edades inferiores a los 25 años. Además, hay que tener en cuenta que estamos hablando de ciclos formativos más bien largos, es decir, que para obtener algún título con validez en el mercado laboral los estudiantes deben aprobar en general más de un curso (dos cursos en el caso de FP de grado superior, cuatro cursos en el grado universitario).

En suma, si entendemos formación permanente (o formación a lo largo de la vida en el lenguaje de la Unión Europea) como aquella formación con cierto grado de formalización, siempre voluntaria, que se realiza principalmente cuando se ha terminado la formación obligatoria, ya en edades avanzadas (como mínimo mayores de 25 años) y que se tiende a compaginar con otras actividades (mayoritariamente el empleo o su búsqueda), por exclusión estaremos hablando de la educación no formal ${ }^{2}$ (No Formal Education -NFE); una forma de enseñanza con objetivos educativos concretos y formalizada, pero con toda la flexibilidad que no tiene la educación formal.

Una de las áreas educativas a la que presta más atención la educación no formal es la formación laboral. Este área comprende los programas para facilitar una formación técnica que permita acceder al empleo y toda la gran variedad de programas y cursos dirigidos a los trabajadores con empleo, incluidas las acciones formativas proporcionadas por las propias empresas para sus trabajadores. Es precisamente la educación no formal protagonizada y dirigida a los trabajadores la que constituye el objeto de estudio de este artículo. Hay que destacar que la atención a este concepto de educación no formal nos va a permitir realizar un análisis de la formación laboral que va más allá de las actividades de formación de las empresas al integrar también las estrategias formativas de los individuos.

\footnotetext{
${ }^{2}$ El término educación no formal se utilizó por primera vez en la International Conference on World Crisis in Education que tuvo lugar en Virginia (USA) en 1967. El documento que sirvió de base para este congreso fue elaborado bajo la supervisión de Philip Coombs por la Oficina de Planeamiento de la Educación de la Unesco que él mismo dirigía. Pero no fue hasta 1975 con la publicación del libro de Ph. Coombs y M. Ahmed cuando se comenzó a delimitar y definir lo que se entendería por educación formal, no formal e informal (Colom Cañellas 2005).
} 


\section{MARCO TEÓRICO. LA FORMACIÓN DE LOS TRABAJADORES COMO FENÓMENO DIFERENCIAL}

Diversas perspectivas teóricas han señalado la importancia de la formación laboral como generadora de cualificaciones profesionales que se traducen a su vez en el logro de mejores condiciones de trabajo para los empleados. El propio modelo teórico del capital humano se inicia con el análisis de la formación en el trabajo antes de abordar la educación formal (Becker 1983). Los beneficios de la formación laboral en las trayectorias profesionales de los asalariados se ha puesto también de manifiesto desde las teorías que podríamos denominar credencialistas (Arrow 1991; Spence 1991; Thurow 1975) hasta los planteamientos teóricos de la tradición de la segmentación de los mercados de trabajo (Doeringer y Piore 1985).

Puesto que la formación laboral constituye un factor muy importante en los logros profesionales de los individuos y en la rentabilidad de las empresas, las investigaciones empíricas se han centrado en determinar las características individuales y organizativas que se relacionan con dicha formación y en su impacto en los resultados empresariales e individuales. A este respecto, los primeros modelos que se dirigen a evaluar la formación se inician en los años 70 si bien es a principios de los 90 cuando comienzan a ser más numerosos (Pineda 2000; Kirkpatrick 1999). Estos modelos se centran sobre todo en el efecto de la formación en la productividad de la empresa, la mejora en la calidad del producto y la rentabilidad de la inversión en formación (Caparrós, Navarro y Rueda 2010).

Entre las características de las empresas que favorecen la formación, el tamaño ha sido una de las más nombradas y analizadas en las investigaciones sobre esta materia. Son las grandes empresas las que más invierten en la formación de sus trabajadores. Las razones citadas para explicar este comportamiento son diversas. Se habla de que tales empresas pueden beneficiarse de economías de escala al conseguir costes más bajos que las pequeñas, tienen una estructura más formalizada o incluso departamentos de formación y sufren una menor rotación laboral al tener mayores posibilidades de retener a los trabajadores formados por medio de salarios más altos y mejores perspectivas de promoción interna que las empresas pequeñas. También disponen de recursos para organizar cursos de manera regular bien en sus propias instalaciones 0 acudiendo a otras empresas de formación (Escobar, Rivière y Glick 2010; Frazis, Gittleman y Joyce 2000; Peraita 2000; Barba, Aragón y Sanz 2000; Lynch y Black 1998; Greenhalgh y Mavrotas 1994; Knoke y Kalleberg 1994). Uno de los efectos a los que puede dar lugar este fenómeno es la polarización de las empresas, con una clara desventaja para las pequeñas y medianas que no pueden disponer de personal cualificado en la misma medida que las grandes. Esta relación del tamaño de la empresa y la formación se daría en el caso de la formación financiada por la empresa. En la formación financiada por el propio trabajador algunas investigaciones no encuentran tal relación (Caparrós, Navarro y Rueda 2009). 
Además del tamaño de la empresa, el sector productivo constituye un elemento explicativo de las diferencias en la incidencia de la formación. En investigaciones referidas a España, se han señalado el sector financiero y los servicios educativos y sanitarios como los sectores en los que aumenta la probabilidad de recibir formación por parte de la empresa (Escobar, Rivière y Glick 2010; Alba Ramírez y Tugores Ques 2000; Peraita 2000). En el lado opuesto se situarían el sector primario, las industrias manufactureras y la hostelería. Parece que en estos sectores las empresas no tienen demasiado interés en formar a sus trabajadores. Las pocas investigaciones que han abordado la formación no financiada por la empresa señalan también a los trabajadores de los sectores de enseñanza y sanidad como los más proclives a formarse, mientras que los pertenecientes a los sectores de transporte y construcción serían los que menos realizan actividades formativas (Caparrós, Navarro y Rueda 2009). Se han señalado asimismo otras características de las empresas relacionadas con una mayor formación; así, esta sería más intensa en industrias expansivas, de rápido crecimiento, innovadoras y sometidas a grandes cambios tecnológicos (Huerta Arribas 2003; Huergo 2002; Green 1993).

Estrechamente relacionado con el tamaño de la empresa, la presencia de sindicatos constituye un factor que favorece la formación de los trabajadores (Dustmann y Schönberg 2009; Lynch y Black 1998; Frazis, Herz y Horrigan 1995). Sin olvidar la propia influencia del marco institucional en el que se configuran las relaciones de empleo en cuanto a derechos de antigüedad, de pensión, sistemas de remuneración y legislación contractual (Knoke y Kalleberg 1994; Greenhalgh y Mavrotas 1994).

Si nos referimos a las características de los trabajadores, el acceso diferencial de hombres y mujeres a la formación ha constituido un resultado frecuente en las investigaciones realizadas al efecto. Así, las mujeres recibirían menor formación por diversos motivos, bien por las propias expectativas de participación en el mercado de trabajo de las mujeres, bien porque las empresas consideran a las mujeres menos estables laboralmente y pueden perder la inversión realizada en ellas, o bien por el tipo de empleos destinados a uno u otro sexo (Jonker y de Grip 1999; Ibáñez Pascual 1999; Green 1993; Booth 1993; Maruani 1993; Lynch 1992; Booth 1991). Sin embargo, investigaciones más recientes no han encontrado diferencias significativas en la probabilidad de recibir formación por parte de la empresa entre hombres y mujeres (Caparrós, Navarro y Rueda 2009; Peraita 2000). Sí habría diferencias a favor de las mujeres en la formación que obedece a la iniciativa de los propios trabajadores y no está financiada por la empresa (Caparrós, Navarro y Rueda 2009). En cuanto a la edad del trabajador, la formación en España tiende a realizarse cuando se está iniciando la vida laboral o en el periodo de pleno desarrollo (35-44 años) —cuando el individuo tiene más capacidad de aprendizaje-, mientras que los trabajadores de mayor edad ven mermadas sus probabilidades de recibir formación. Estos trabajadores con pocas posibilidades de recualificación corren el riesgo, si son despedidos de 
la empresa, de verse excluidos del mercado de trabajo (Planas y Rifá 2001; Peraita 2000).

De las características de los trabajadores, el nivel educativo inicial ha sido considerado básico en la explicación de la provisión de formación. Son los trabajadores con estudios universitarios los que más formación reciben por parte de la empresa. Se forma, por tanto, a quienes ya tienen altas cualificaciones y pueden aprovechar mejor la inversión realizada en formación (Escobar, Rivière y Glick 2010; Tugores y Alba 2002; Peraita 2000; Altonji y Spletezer 1991). Incluso se han observado diferencias según el tipo de formación. La de carácter más formal estaría dirigida a los niveles medios y superiores de la empresa, mientras que en los empleados de puestos más bajos el aprendizaje se realizaría fundamentalmente en el propio puesto de trabajo (Martín Artiles y Lope 1999). El nivel educativo también presenta una relación positiva con la formación no financiada por la empresa; la probabilidad de que un trabajador adquiera formación laboral por su cuenta aumenta con el nivel educativo alcanzado (Caparrós, Navaro y Rueda 2009).

Como señala Planas y Rifá (2001) la falta de formación inicial puede constituir, por tanto, un factor de exclusión. Las empresas tienden a formar a los trabajadores más cualificados con el consiguiente riesgo de polarización de las trayectorias formativas y laborales; o en otras palabras, la formación laboral reproduce las desigualdades de partida dando lugar a un círculo "virtuoso" en las inversiones en capital humano: los que más invierten en formación son a su vez los que más reciben formación por parte de la empresa (Escobar, Rivière y Glick 2010; Peraita 2000). Como ya se ha señalado, esta inversión en los más cualificados se ha observado asimismo en otros tipos de formación, como es aquella que no está financiada por la empresa y obedece sobre todo a la iniciativa individual. Se puede afirmar, por tanto, que determinados individuos desarrollan estrategias formativas de acumulación de capital humano favorecidas por las propias empresas.

Estos resultados son especialmente relevantes si tenemos en cuenta los logros laborales que se asocian a la formación. Los trabajadores más cualificados y que acumulan más formación ocupan puestos elevados en la jerarquía ocupacional, tienen mayores posibilidades de promoción, reciben más altos salarios y suelen desarrollar trayectorias laborales y salariales ascendentes (Rueda 2006; Frazis y Loewenstein 2005; Albert, García Serrano y Hernanz 2005; Peraita 2000; García Espejo 1999; Kider y Rouiller 1997; Kirkpatrick 1997). Respecto a los salarios, en España algunas investigaciones han encontrado una relación directa entre la formación y los niveles salariales de los trabajadores más cualificados (Mato 2002; Alba-Ramírez 1994). También en España se han encontrado diferencias salariales por género según el tipo de formación. En los hombres la formación financiada por la empresa supone un incremento salarial que se reduce en el caso de la formación autofinanciada. Por el contrario, en las mujeres es precisamente este último tipo de formación la que ejerce un efecto positivo en sus salarios. Se ha argumentado que tal resultado podría expli- 
car por qué las mujeres tienen una presencia mayor en la formación autofinanciada (Caparrós, Navarro y Rueda 2009).

Un logro laboral muy relevante es la mayor estabilidad en el empleo. A este respecto, investigaciones internacionales han observado que la formación está muy asociada a las situaciones de estabilidad laboral (Frazis, Herz y Horrigan 1995; Knoke y Kalleberg 1994). Al igual que en otros países, en España se observa asimismo una asociación positiva entre la formación y la permanencia del trabajador en la empresa (Caparrós, Navarro y Rueda 2004; Sáez 2002; Barba, Aragón y Sanz 2000), un efecto muy importante si tenemos en cuenta la elevada temporalidad del empleo en nuestro país. Las empresas, por tanto, destinan la inversión formativa a aquellos trabajadores con contrato fijo y más antigüedad en la organización para asegurarse así que la inversión efectuada no se pierda por el abandono de la empresa. A la inversa, también se ha observado en nuestro país que la probabilidad de invertir en formación no financiada por la empresa es mayor entre los trabajadores temporales y con menor antigüedad (Caparrós, Navarro y Rueda 2004).

\section{Metodología de ANÁLISIS}

La base de datos utilizada es la Encuesta sobre la Participación de la Población Adulta en las Actividades de Aprendizaje (para la ficha técnica de la muestra y el cuestionario ver Instituto Nacional de Estadística 2008b). En la presente investigación, el tamaño de la muestra es de 8354 asalariados, de los cuales un 33,5\% han participado en algún tipo de educación no formal.

La unidad de análisis serán los cursos que han realizado los encuestados (en total 3962). Cada curso se entiende como "un evento", en el sentido de decisiones de formación de los sujetos. Nuestra muestra será de eventos repetidos, ya que en el cuestionario se recoge información de hasta un máximo de tres cursos, por lo que cada sujeto contará con tres eventos ${ }^{3}$, que pueden ser de formación o de no formación (panel balanceado), y las variables que caracterizan a los sujetos se repetirán las tres veces. En este diseño de la matriz de datos es muy probable que aquellos cursos realizados por la misma persona mantengan alguna relación, por lo que, para asegurar que los errores no correlacionan (heterogeneidad no observada), el modelo estadístico utilizado es de medidas repetidas (repeated measures), en concreto un modelo de efectos aleatorios ${ }^{4}$.

\footnotetext{
${ }^{3} \mathrm{Si}$ la persona entrevistada participa en más de tres a lo largo de los doce meses previos a la entrevista se seleccionan aleatoriamente tres de estas actividades.

${ }^{4}$ En los modelos con estructura de datos de panel se ha de elegir entre modelos de efectos fijos o aleatorios.
} 
La construcción de las variables dependientes es producto de los propios objetivos de la investigación y de las posibilidades de la encuesta. En concreto se ha utilizado el módulo de la encuesta que aborda la 'Educación no formal' y se han elaborado cuatro variables dependiente dicotómicas (cumplir la condición versus resto de situaciones): 'Formación financiada por la empresa y realizada por motivos relacionados con el trabajo' (1856 cursos), 'Formación en el puesto' (983 cursos), 'Formación no financiada por la empresa y realizada por motivos relacionados con el trabajo' (569 cursos) y 'Formación no financiada por la empresa y realizada por motivos no relacionados con el trabajo' (547 cursos).

Las variables independientes de los cuatro modelos son las mismas. Variables referidas a "características sociodemográficas": sexo, edad (reagrupada en décadas hasta 55 y más años), nacionalidad, nivel de estudios, y si el trabajador está realizando algún tipo de formación dentro del sistema educativo reglado. Variables referidas a las "características del trabajo": la categoría ocupacional, el tipo de jornada (a tiempo completo o a tiempo parcial), la antigüedad del trabajador en la empresa, y si el contrato es temporal o indefinido. "Variables relativas a la empresa": la rama de actividad y su tamaño.

\section{FORMACIÓN LABORAL DE LOS TRABAJADORES. TIPOS Y PERFILES}

El porcentaje de asalariados por tipos de formación figura en la Tabla 1. Como puede observarse un $33,5 \%$ de los asalariados ha realizado algún tipo de aprendizaje no formal. Un $16,7 \%$ ha recibido formación financiada por la empresa y llevada a cabo por motivos laborales y un $9,1 \%$ de los asalariados han recibido formación en el puesto de trabajo. La formación no pagada por la empresa pero que tiene una motivación laboral afecta al 6,3\% de los asalariados y se observa una misma proporción en el caso de aquellos cuya formación no ha sido financiada por la empresa ni se ha desarrollado por motivos laborales. En cuanto a la duración de los cursos, cuando la formación es pagada por la empresa los cursos son de menor duración, especialmente la que se lleva a cabo en el propio puesto de trabajo. Los cursos más largos son aquellos cuya financiación no corre a cargo de la empresa ni se realizan por motivos laborales.

Para los cuatro tipos de formación señalados, se han elaborado modelos de regresión que se espera presenten características distintivas tanto en las variables asociadas a la dimensión personal de los asalariados, como a las características de los puestos y de las empresas. Los coeficientes de los modelos expresados en odds ratio y su significación se encuentran en la Tabla 2.

En nuestro caso se ha elegido el modelo de efectos aleatorios especialmente teniendo en cuenta los objetivos del estudio, el tipo de datos con los que se cuenta y el tamaño de la muestra (Mayorga 2000:9; Baltagi 1995). 
Tabla 1.

Porcentaje de asalariados por tipo de cursos y duración de los cursos.

\begin{tabular}{c|c|c|c|c|c}
\hline N. ${ }^{\circ}$ de asalariados: 8354 & $\begin{array}{c}\text { todos los } \\
\text { cursos } \\
\text { aprendizaje } \\
\text { no formal }\end{array}$ & $\begin{array}{c}\text { coste } \\
\text { empresa } \\
\text { y motivos } \\
\text { laborales }\end{array}$ & $\begin{array}{c}\text { coste no } \\
\text { empresa y } \\
\text { motivos } \\
\text { laborales }\end{array}$ & $\begin{array}{c}\text { coste no } \\
\text { empresa y } \\
\text { no motivos } \\
\text { laborales }\end{array}$ & $\begin{array}{c}\text { formación } \\
\text { puesto }\end{array}$ \\
\hline Asalariados participantes (\%) & 33,5 & 16,7 & 6,3 & 6,3 & 9,1 \\
\hline \multicolumn{7}{c|}{ Duración de los cursos (\% sobre filas) } \\
\hline Hasta 10h & 24,7 & 27,3 & 14,2 & 10,2 & 33,9 \\
\hline De 11 a 20h. & 18,3 & 19,6 & 14,9 & 11,0 & 22,2 \\
\hline De 21 a 40h. & 24,7 & 27,3 & 24,1 & 19,0 & 23,4 \\
\hline De 41 a 60h. & 9,5 & 8,2 & 12,3 & 14,6 & 7,4 \\
\hline De 61 a 80h. & 7,1 & 6,1 & 8,1 & 14,1 & 4,7 \\
\hline Más de 80h. & 15,6 & 11,4 & 26,4 & 31,1 & 8,4 \\
\hline N de cursos & 3962 & 1856 & 569 & 547 & 983 \\
\hline
\end{tabular}

Fuente: EADA 2007. Elaboración propia.

\section{Formación financiada por la empresa y realizada por motivos relacionados con el trabajo}

Si analizamos las características demográficas de los trabajadores que más se asocian con la formación financiada por la empresa a través de cursos y programas formativos, lo primero que hay que señalar es que no se aprecian diferencias significativas entre hombres y mujeres en la recepción de formación. Por tanto, la creencia de que las mujeres tienen mayores probabilidades de abandono de la actividad laboral —una creencia a la que se suele imputar su trato desigual ante la formación por el miedo a la pérdida de la inversión realizada por la empresa - no parece que sea una idea que predomine en la actualidad dentro del sector empresarial, y la distribución por género de los trabajadores que reciben formación es mucho más igualitaria.

Son los trabajadores más jóvenes, entre 25 y 34 años, los que más probabilidades tienen de ser receptores de la formación, mientras que los trabajadores de más de 50 años presentan muy pocas posibilidades de ser formados por la empresa. Junto a los más jóvenes, la formación también está dirigida a los que poseen un mayor capital humano inicial, los trabajadores universitarios; estos empleados tienen una probabilidad de recibir formación cuatro veces superior a la de los trabajadores con estudios primarios. Se forma, por tanto a los más formados, los que pueden aprovechar en mayor medida la inversión efectuada por la empresa. Este resultado junto con el comportamiento de la variable anterior convierte a los trabajadores de mayor edad y menos cualificados en un 
colectivo con serios problemas de recualificación que puede verse excluido del mercado de trabajo en caso de pérdida de empleo.

Si nos referimos a las características del puesto, la formación financiada por la empresa es más intensa en las categorías ocupacionales que requieren altas cualificaciones: profesionales y técnicos de grado superior, seguidos de técnicos de grado medio. Estos colectivos tienen más del doble de probabilidades de asistir a cursos de formación que los trabajadores no cualificados; un fenómeno que puede incrementar asimismo la desigualdad en el mercado de trabajo al ampliarse la distancia entre los más y los menos cualificados. La antigüedad en la empresa, una proxy de la experiencia acumulada en el puesto, también resulta una variable significativa. Las empresas no destinan la formación a los trabajadores que llevan menos de tres años en la empresa y que tienen contratos temporales, una forma de intentar evitar la pérdida de la inversión realizada en formación. Las empresas dirigen su inversión formativa a los trabajadores con contrato fijo y que tienen más antigüedad; unas pautas similares a las registradas en investigaciones de carácter internacional. Si la jornada laboral es a tiempo completo, los trabajadores tienen tres veces más probabilidades de acudir a cursos organizados por la empresa que los que están contratados a tiempo parcial. En definitiva se forma a los trabajadores que presentan los lazos más firmes con la organización.

En cuanto a las características de las empresas proveedoras de formación, son las de mayor tamaño, que suelen ser intensivas en tecnología y cuentan con un elevado número de puestos cualificados, las que más forman a sus empleados; un resultado común al conjunto de investigaciones que se han realizado al efecto. Junto al tamaño de la empresa cabe citar el sector de actividad, destacando el sector financiero; un sector que requiere personal con altas cualificaciones. A este sector le sigue el de otros servicios, integrado por actividades sociales, servicios prestados a la comunidad y servicios personales.

\section{Formación en el puesto de trabajo}

Otra modalidad de formación proporcionada por la empresa es el aprendizaje que se adquiere en el puesto de trabajo. En este tipo de formación sí que se observan diferencias acusadas según el género. Los hombres tienen mayores probabilidades de adquirir experiencia y conocimientos en el propio puesto de trabajo. La razón de este resultado podría estar en la segregación ocupacional por género, de forma que en los empleos ocupados mayoritariamente por hombres sería más frecuente la adquisición de habilidades en el propio puesto.

No se aprecian diferencias por edad que sean estadísticamente significativas, pero sí por estudios. Nuevamente son los más educados los que más se forman, pero ahora van a ser los trabajadores con un nivel educativo de Formación Profesional Reglada, sobre todo en los niveles más altos, los que presentan mayores probabilidades de adquirir la formación por medio de un aprendizaje en el puesto de trabajo. En relación con este resultado la categoría ocupacional que más se asocia con este tipo de formación es la de 
técnicos de grado medio, seguida por profesionales y técnicos superiores y trabajadores de servicios y comercio. La antigüedad en la empresa no resulta muy significativa, si bien se aprecia una menor propensión a que se proporcione este tipo de formación en los trabajadores que llevan menos de un año en la empresa. Ni el tipo de contrato ni la modalidad de jornada laboral resultan variables significativas.

El efecto del tamaño de la empresa es incluso superior que en el tipo de formación a través de cursos. Las empresas con más de 50 trabajadores tienen una probabilidad casi cuatro veces superior de formar a sus trabajadores en el propio puesto de trabajo que las más pequeñas. Se trata además de empresas pertenecientes sobre todo al sector financiero, seguidas de aquellas enmarcadas en las ramas de educación y administración pública. La industria y el comercio también resultan sectores significativos.

\section{Formación no financiada por la empresa y realizada por motivos relacionados con el trabajo}

Los trabajadores también pueden formarse por propia iniciativa, asumiendo ellos los costes de la formación. Aquí son las mujeres las que tienen mayores probabilidades de realizar esta inversión por su cuenta y también los empleados más jóvenes. Asimismo cuanto mayor es el nivel educativo mayor es la iniciativa de acumular capital humano no financiado por la empresa. En concreto los titulados universitarios tienen una probabilidad casi cinco veces superior de formarse que los trabajadores con estudios primarios. Se puede afirmar, por tanto, que los trabajadores con mayor educación formal desarrollan estrategias acumulativas de capital humano, favorecidas, como hemos visto, por las propias empresas. Este resultado acentúa aún más la distancia entre los itinerarios formativos de los trabajadores a lo largo de la vida laboral, y viene a ser un rasgo en el que nuestro país sobresale respecto a otros países europeos (Mato 2010).

Respecto a las características del puesto, estos trabajadores suelen tener una categoría laboral de técnico de grado medio o de trabajadores de servicios y comercio, realizan su actividad laboral a jornada completa pero presentan una relación temporal con la empresa. Se trata, por tanto, de empleados con vínculos débiles con la organización que pueden tratar de estabilizarse y mejorar sus perspectivas de empleo y promoción realizando este tipo de inversiones formativas. El tamaño de la empresa no constituye una variable tan significativa como en los tipos de formación anteriores y claramente destacan dos ramas de actividad: sanidad y educación.

\section{Formación no financiada por la empresa y realizada por motivos no relacionados con el trabajo}

En este artículo también se ha querido analizar la situación de aquellos trabajadores que acuden a recibir formación no financiada por la empresa y que obedece a motivos que a su juicio no están relacionados con el trabajo que desarrollan. Aquí nos encontramos con un mayor número de mujeres y ante los empleados más jóvenes. El nivel de estudios 
Tabla 2.

Modelos de regresión logística de los cuatro tipos de educación no reglada. Odds ratio y significación.

\begin{tabular}{|c|c|c|c|c|}
\hline & $\begin{array}{c}\text { Coste empresa y } \\
\text { motivos laborales } \\
\text { Odds ratio }\end{array}$ & $\begin{array}{l}\text { Formación } \\
\text { puesto } \\
\text { Odds ratio }\end{array}$ & $\begin{array}{c}\text { Coste no empresa } \\
\text { y motivos laborales } \\
\text { Odds ratio }\end{array}$ & $\begin{array}{c}\text { Coste no empresa y } \\
\text { no motivos laborales } \\
\text { Odds ratio }\end{array}$ \\
\hline Ser hombre & ,96 & $1,62^{\text {***}}$ &, $59 * * *$ &, $54^{* * *}$ \\
\hline \multicolumn{5}{|l|}{ Edad (Ref. 25-34) } \\
\hline - Entre 35 y 44 años & ,98 & 1,03 & 1,06 &, $73^{\star *}$ \\
\hline - Entre 45 y 54 años &, 93 & 0,94 &, $64^{* *}$ & ,79 \\
\hline - 55 y más años &, 50 *** &, 71 &, $43^{\star * *}$ &, $53^{* *}$ \\
\hline Ser español & $3,18^{* * *}$ & 1,30 & $2,01^{\star *}$ & ,82 \\
\hline \multicolumn{5}{|c|}{ Nivel de estudios (Ref. Primarios y menos) } \\
\hline - EGB y ESO & $1,82^{* * *}$ & $1,80^{* * *}$ & 1,34 & 1,27 \\
\hline - FP1 & $2,10^{* * *}$ & $2,33^{* * *}$ & $2,84^{* * *}$ & $3,14^{* * *}$ \\
\hline - Bachiller & $3,58^{* * *}$ & $2,00^{* * *}$ & $3,12^{* * *}$ & $2,45^{* * *}$ \\
\hline - FP 2 y 3 & $2,90^{* * *}$ & $2,89^{* * *}$ & $3,62^{* * *}$ & $2,80^{\star * *}$ \\
\hline - Universitarios & $4,81^{* * *}$ & $2,09^{* * *}$ & $4,87^{* * *}$ & $2,58^{* * *}$ \\
\hline \multicolumn{5}{|c|}{ Categoría ocupacional (Ref. No Cualificados) } \\
\hline - Prof. y técnicos superiores & $2,60^{* * *}$ & $2,15^{* *}$ & $2,36^{\star *}$ &, $59^{\star *}$ \\
\hline - Técnicos de grado medio & $2,48^{* * *}$ & $2,81^{* * *}$ & $3,02^{* * *}$ & 66 \\
\hline - Empleados de oficina & $1,46^{*}$ & $1,66^{*}$ & $2,15^{\star *}$ &, $66^{*}$ \\
\hline - Trabaj. de servicios y comercio & $1,50 * *$ & $1,98^{* *}$ & $2,99 * * *$ & ,74 \\
\hline - Cuali. de la industria (y agricul.) & $1,52^{* *}$ & 1,36 & $1,85^{*}$ &, $71^{*}$ \\
\hline \multicolumn{5}{|c|}{ Rama de actividad (Ref. Actividades del hogar) } \\
\hline - Agricultura y pesca & 2,37 & $4,15^{*}$ & 1,09 & ,96 \\
\hline - Industria & 1,97 & $4,12^{*}$ & 1,86 & 1,81 \\
\hline - Construcción & $2,82^{*}$ & 2,56 & 1,83 & 1,71 \\
\hline - Comercio & 2,00 & $4,39 *$ & 1,79 & $2,14^{*}$ \\
\hline - Hostelería & 2,31 & 2,80 & 3,70 & $2,94^{* * *}$ \\
\hline - Transporte & $2,63^{*}$ & 3,38 & 1,78 & $2,48^{* *}$ \\
\hline - Banca & $3,86^{* *}$ & $10,87^{\star * *}$ & 2,97 & $2,11^{*}$ \\
\hline - Inmobiliaria & 2,21 & 1,96 & 2,07 & $2,74^{\star *}$ \\
\hline - Administración & $2,98^{* *}$ & $5,32^{* *}$ & 3,67 & 1,86 \\
\hline - Educación & 2,09 & $9,06^{* * *}$ & $5,70^{* *}$ & $2,54^{\star *}$ \\
\hline - Sanidad & $2,56^{*}$ & $4,66^{*}$ & $5,18^{* *}$ & 1,82 \\
\hline - Servicios personales y otros & $3,78^{* *}$ & 3,83 & 1,91 & $2,01^{*}$ \\
\hline \multicolumn{5}{|c|}{ Tamaño de la empresa (Ref, entre 1 y 5 trabajadores) } \\
\hline - De 6 a 10 trabajadores & $1,60^{* *}$ & ,93 & $1,70^{*}$ & ,74 \\
\hline - De 11 a 19 & 1,25 & $1,59^{*}$ & 1,18 &, 81 \\
\hline - De 20 a 49 & $1,65^{\star * *}$ & $1,99^{* * *}$ & 1,18 & ,98 \\
\hline - 50 y más & $2,23^{* * *}$ & $3,92^{* * *}$ & $1,57^{*}$ & ,88 \\
\hline - No sabe, pero más de 10 & $2,22^{* * *}$ & 1,30 & 1,36 &, $48^{*}$ \\
\hline \multicolumn{5}{|c|}{ Antigüedad en la empresa (Ref, más de 12 años) } \\
\hline - Hasta un año &, $75^{*}$ & $67^{*}$ & 1,20 & 95 \\
\hline - De 2 a 3 años &, $76^{*}$ & ,76 & 1,43 & 1,04 \\
\hline - De 4 a 6 años & 86 & 88 & 1,11 & ,88 \\
\hline - De 7 a 12 años &, 94 &, 77 &, 81 & ,87 \\
\hline Contrato fijo (Ref. Temporal) & $1,30^{* *}$ & 1,05 &, $51^{\star \star \star}$ &, $73^{* *}$ \\
\hline Jornada completa (Ref. TP) & $2,99^{* * *}$ & 1,22 & $1,66^{* *}$ &, 98 \\
\hline Compatibiliza con Ed. Formal &, $61^{* * *}$ &, $36^{* * *}$ &, 76 & $2,25^{\star * \star}$ \\
\hline $\mathrm{N}=24.327 \quad$ Log likehood & $-5980,0343$ & $-3812,7086$ & $-2510,1945$ & $-2687,8828$ \\
\hline
\end{tabular}

Fuente: EADA 2007. Elaboración propia. * $p<0,100 ;{ }^{* *} p<0,050 ;{ }^{* * *} p<0,010$. 
no presenta un comportamiento tan progresivo como en los tipos formativos anteriores, aunque los que desarrollan estas actividades poseen un nivel educativo superior en todos los casos al de estudios primarios. Un dato especialmente relevante es que los trabajadores que están todavía dentro del sistema educativo reglado son los que más probabilidades tienen de participar en actividades formativas relacionadas con intereses ajenos a los laborales. Se trata, por tanto, de una formación realizada en gran medida por trabajadores que compatibilizan trabajo y estudios; una situación que no se observa en los otros tipos de formación.

Además, la categoría profesional que mayor asociación presenta es la de trabajador no cualificado con una modalidad de contrato temporal, lo que sugiere, junto con el resultado anterior, que nos encontramos ante jóvenes inmersos todavía en la educación formal y que realizan alguna actividad laboral. En la misma línea de estos resultados apuntan las características de las empresas donde trabajan. Así, es más probable que la empresa sea de pequeño tamaño y perteneciente sobre todo al sector de la hostelería.

\section{LOS QUE NO SE FORMAN. RAZONES Y PERFILES}

Como se señalaba en la introducción, un aspecto poco analizado de la educación de adultos y del acceso diferencial que los trabajadores tienen a ella es el que se refiere a los que no se forman, las razones para no hacerlo y en general las dificultades que sufren los asalariados a la hora de poder participar en estas actividades. La EADA nos ofrece una nueva fuente de información con interesantes matices al separar a los que no tuvieron intención de formarse de los que manifestaron ese deseo, y al permitirnos profundizar además en las razones de cada uno de los dos grupos para no formarse. Junto a este análisis, en el presente apartado se lleva a cabo el estudio del perfil sociodemográfico y ocupacional por medio de regresiones logísticas de aquellos que, deseando formarse, no han podido hacerlo.

Como ya hemos visto un $33,5 \%$ de los asalariados han realizado algún tipo de educación no formal en el año de referencia, quedando un grupo mayoritario de más del $60 \%$ que no ha pasado por ningún tipo de formación, sea formal o no formal. Este resultado sugiere que queda mucho por recorrer en cuanto a la sensibilización de los asalariados ante la necesidad de la formación continua, pues la mayoría no desearon formarse, mientras que el $8 \%$ querían hacerlo pero por alguna razón no pudieron.

Las razones por las que estos trabajadores no desearon formarse 0 no pudieron hacerlo se organizan de dos maneras (ver Tabla 3). El cuestionario EADA aborda estos motivos a partir de una batería de preguntas dicotómicas, permitiendo que las respuestas sean múltiples (columnas A de la tabla); posteriormente se pide al encuestado que señale qué motivo de ellos es el principal (columnas B). Los dos grupos presentan ciertas semejanzas pues los asalariados no se forman debido, o bien a las responsabilidades familiares, o bien a la dificultad de compatibilizar los tiempos de trabajo con el 
horario de los cursos. Como se aprecia, ambas razones coinciden en la dificultad de gestionar los tiempos de formación y en muchos casos se solapan, por ejemplo un $44 \%$ de los que esgrimen razones familiares también aducen problemas con el horario laboral. Este hecho nos permite adelantar que las medidas para aumentar la formación de los trabajadores tienen que pasar por facilitarla dentro del horario laboral o permitir que la formación sea compatible con el horario de trabajo. Además, que la falta de tiempo sea el motivo mayoritario en ambos grupos introduce la duda sobre la diferencia real entre ellos, pues los primeros, frente a la dificultad material de encontrar tiempo para la formación, pueden optar por no desearla (ni planteársela), una estrategia muy humana para evitar la frustración (tabla 3).

Pese a las similitudes, el grupo de los que no desearon formarse presenta dos particularidades a resaltar. Por una parte, el $10,5 \%$ no ofrece ninguna razón para no formarse, dato que puede estar asociado a que algunos trabajadores "ni se planteen los motivos". Por otra parte, un 19\% entienden que "no lo necesitan". Estas respuestas reflejan una visión de la formación alejada del espíritu de "La formación a lo largo de la vida" que están defendiendo las instituciones públicas que pretenden que la formación continua no sea tanto una cuestión de necesidad como de oportunidad; es decir, en un mundo de innovación, el discurso de la formación permanente implica que los trabajadores se entiendan a sí mismos como "recurso" o "capital humano" y que de esa forma gestionen su formación.

Tabla 3.

Motivos de no formación

\begin{tabular}{lrrrr}
\hline & No desearon & \multicolumn{2}{r}{ No pudieron } \\
\hline & $\mathrm{A}$ & \multicolumn{1}{c}{$\mathrm{B}$} & $\mathrm{A}$ & $\mathrm{B}$ \\
\hline Yo no reunía los requisitos previos & 1,1 & 3,9 & 7,1 & 5,3 \\
\hline La formación era demasiado cara/no me lo podía permitir & 2,3 & 2,0 & 13,6 & 16,5 \\
\hline Por falta de apoyo del empleador & 1,8 &, 5 & 7,9 & 1,6 \\
\hline No era compatible con el horario de trabajo & 17,4 & 9,6 & 46,9 & 23,7 \\
\hline No disponía de tiempo por responsabilidades familiares & 32,4 & 25,9 & 38,6 & 25,8 \\
\hline Las actividades no tenían lugar a una distancia razonable & 2,7 & 1,0 & 9,0 & 3,6 \\
\hline No estaba seguro/a de querer volver a algo parecido al colegio & 7,2 & 5,7 & 2,5 &, 3 \\
\hline La edad o la salud & 4,2 & 2,6 & 3,0 & 1,6 \\
\hline La formación exigía una matrícula completa en un ciclo de FP &, 4 &, 1 & 2,6 &, 1 \\
\hline La formación exigía matrícula completa en un módulo de FP &, 3 &, 1 & 1,8 &, 1 \\
\hline No lo necesitaba para el trabajo & 19,4 & 11,4 & & \\
\hline No lo necesitaba por motivos personales (no relac. con el trabajo) & 10,7 & 7,6 & & \\
\hline Otros & 23,1 & 19,1 & 24,0 & 21,4 \\
\hline Ninguna razón & 10,5 & 10,5 & & \\
\hline TOTAL & $\mathrm{N}=4631$ & 100,0 & $\mathrm{~N}=720$ & 100,0 \\
\hline
\end{tabular}

Fuente: AEDA 2007. Elaboración propia. Las columnas A reflejan las respuestas múltiples, por ello los porcentajes no suman 100. En las B se selecciona la razón más importante (suma 100). 
El último objetivo de este apartado es aclarar el perfil de aquellos trabajadores que han manifestado expresamente su deseo de formarse y su imposibilidad para hacerlo (valor 1 en la variable dependiente de la regresión Logit (684 persona) frente a los que sí pudieron (valor 0). Este será el modelo 1; en los modelos 2 y 3 (todos recogidos en la tabla 4) se selecciona de este colectivo a aquellos asalariados que no han podido formarse según las razones mayoritarias: por incompatibilidad con el horario de trabajo (322 personas) y por no disponer de tiempo por las obligaciones domésticas (263 personas). Las variables independientes son las mismas que las incorporadas en los modelos del apartado anterior.

Los asalariados con mayores probabilidades de tener dificultades para formarse se encuentran en las franjas de edades centrales, especialmente entre los 35 y 45 años, época en la biografía personal asociada a la crianza de hijos pequeños y seguramente a la exigencia de fuertes compromisos para la construcción de la carrera laboral. Los trabajadores no nacionales muestran una dificultad significativamente mayor, efecto que también se observa entre los que tienen EGB, secundaria obligatoria y FP1. En cuanto a las características laborales, las probabilidades de tener mayores dificultades para formarse son mayores en los trabajadores cualificados, en aquellos que trabajan en microempresas, con una antigüedad menor a 12 años y a jornada completa. En conjunto, las características diferenciadoras de los que no pudieron formarse reflejan una posición "inferior" a la de aquellos que lo han hecho. Si, como hemos visto, la probabilidad de formarse está más asociada a niveles de formación medios y sobre todo superiores, y a categorías ocupacionales de profesionales y técnicos, esta posición intermedia nos habla de asalariados con estudios inmediatamente superiores a los primarios obligatorios y con categorías de trabajadores manuales, pero cualificados, es decir, que están en una posición laboral en la que la formación sí se valora, pero sin embargo las características de los empleos, de las empresas y la propia organización laboral dificultan esta actividad.

El argumento de la organización laboral es especialmente congruente para los casos en los que la razón aducida es la dificultad de compatibilizar la formación con el horario de trabajo (Modelo 2). En este caso, las características diferenciadoras se refieren mayoritariamente a las características laborales, en concreto tienen este tipo de impedimento de forma mayoritaria los trabajadores cualificados, tanto de la industria como de los servicios, y aquellos que trabajan en la microempresa.

En el perfil de los que no se forman por problemas de conciliación las características personales juegan una importancia significativa. El grupo con mayor probabilidad es el de las mujeres de entre 35 y 44 años y, aunque el nivel de estudios no diferencia mucho a la población, sí padecen mayor dificultad aquellos con nivel de estudio de FP1. La rama de actividad también es explicativa en el modelo (ayuda a mejorar su ajuste) $y$, aunque las diferencias entre las actividades no logran la significación estadística, el peso de sus coeficientes nos indica que es en la rama de referencia, la de "actividades 


\section{Tabla 4.}

Modelos de regresión logística. No pudieron formarse y razones fundamentales.

Modelos 1-3. Odds ratio y significación.

\begin{tabular}{|c|c|c|c|}
\hline & $\begin{array}{c}\text { No pudieron formarse } \\
\text { MODELO } 1 \\
\text { Odds ratio }\end{array}$ & $\begin{array}{l}\text { Horario laboral } \\
\text { MODELO } 2 \\
\text { Odds ratio }\end{array}$ & $\begin{array}{c}\text { Conciliación doméstica } \\
\text { MODELO } 3 \\
\text { Odds ratio }\end{array}$ \\
\hline Ser hombre & 98 & 1,12 &, $66^{* *}$ \\
\hline Edad (Ref. 25-34) & ** & & \\
\hline - Entre 35 y 44 años & $1,35^{\star \star \star}$ & $1,31^{*}$ & $1,82^{\star * *}$ \\
\hline - Entre 45 y 54 años & $1,26^{*}$ & 1,15 & 1,32 \\
\hline - 55 y más años & 1,30 &, 81 & ,82 \\
\hline Ser español &, $43^{\star \star \star}$ &, $43^{\star \star \star}$ &, $33^{\star \star \star}$ \\
\hline Nivel de estudios (Ref. a Primarios y menos) & $*$ & & ** \\
\hline - EGB y ESO & $1,59^{\star *}$ & 1,23 & 1,16 \\
\hline$-F P 1$ & $1,50^{\star *}$ & 94 & $1,72^{* *}$ \\
\hline - Bachiller & 1,24 & 1,07 & 1,19 \\
\hline$-F P 2$ y 3 & 1,08 & 1,11 & ,91 \\
\hline - Universitarios & 1,30 & 1,15 & 83 \\
\hline Categoría ocupacional (Ref. No Cualificados) & $* \star *$ & $* * *$ & \\
\hline - Profe. Y técnicos superiores & $1,47^{\star *}$ & $1,72^{\star *}$ & 1,20 \\
\hline - Técnicos de grado medio & $1,62^{* *}$ & $1,73^{*}$ & 1,35 \\
\hline - Empleados de oficina & $1,58^{* *}$ & $2,16^{* *}$ & 1,44 \\
\hline - Traba. de servicios y comercio & $2,18^{* * *}$ & $3,53^{\star \star *}$ & 1,49 \\
\hline - Cuali. de la industria (y agricul.) & $2,60^{\star \star *}$ & $3,75^{\star \star *}$ & 1,34 \\
\hline Rama de actividad (Ref. Actividades del hogar) & & & ** \\
\hline - Agricultura y pesca & $2,15^{\star *}$ & $2,27^{*}$ & 2,22 \\
\hline - Industria & 1,36 & 1,12 & 1,89 \\
\hline - Construcción & 1,55 & 1,09 & 1,49 \\
\hline - Comercio & 1,06 & ,95 & 89 \\
\hline - Hostelería & 1,29 & 1,31 & 1,45 \\
\hline - Transporte & 1,33 & 1,23 & 1,15 \\
\hline - Banca &, 52 & ,39 &, 39 \\
\hline - Inmobiliaria & 1,23 & 1,31 & 1,72 \\
\hline - Administración & 1,04 & 1,10 &, 56 \\
\hline - Educación & 1,21 & ,98 & 75 \\
\hline - Sanidad & 1,23 & 1,69 & 1,53 \\
\hline - Servicios personales y otros & 1,10 & ,78 & 1,52 \\
\hline Tamaño de la empresa (Ref. entre 1 y 5 trabajadores) & $* * *$ & *** & *** \\
\hline - De 6 a 10 trabajadores &, $75^{*}$ & , $67^{*}$ & 85 \\
\hline - De 11 a 19 &, $59^{\star \star *}$ &, $47^{\star \star \star}$ & 66 \\
\hline - De 20 a 49 &, $42^{\star \star \star}$ &, $36^{\star \star *}$ &, $49^{\star * *}$ \\
\hline-50 y más &, $43^{* \star *}$ &, $31^{* * *}$ &, $38^{* * *}$ \\
\hline - No sabe, pero más de 10 &, $25^{\star \star *}$ &, $15^{* \star *}$ &, $43^{*}$ \\
\hline Antigüedad en la empresa (Ref. a más de 12 años) & & +2 & \\
\hline - Hasta un año & $1,55^{* *}$ & $1,57^{*}$ & ,90 \\
\hline - De 2 a 3 años & $1,56^{\star \star \star}$ & 1,26 & 85 \\
\hline - De 4 a 6 años & $1,55^{\star \star \star}$ & 1,33 & $1,47^{*}$ \\
\hline - De 7 a 12 años & $1,53^{\star \star *}$ & $1,49^{\star *}$ & 1,07 \\
\hline Contrato fijo (Ref. Temporal) &, 90 & 88 & ,94 \\
\hline Jornada completa (Ref. Tiempo Parcial) & $1,86^{* * *}$ & 1,28 & $2,54^{* \star *}$ \\
\hline 2 I galikelihend & $\mathrm{N}=3.897$ & $\mathrm{~N}=3.558$ & $\mathrm{~N}=3.527$ \\
\hline 2 Log IIKellnood & 3264,082 & 1926,793 & 1643,965 \\
\hline
\end{tabular}

Fuente: EADA 2007. Elaboración propia. ${ }^{*} p<0,100 ;{ }^{* *} p<0,050 ;{ }^{* \star *} p<0,010$. 
del hogar", donde se presentan mayores dificultades por razones domésticas para la formación. Otras características estructurales asociadas a las dificultades de formación por razones familiares son que las empresas sean de tamaño menor a 20 trabajadores y que estos estén empleados a jornada completa.

Un dato a destacar en los tres modelos es la falta de significatividad de tener un contrato fijo o temporal. Parece que el tipo de contrato no influye sobre las dificultades de formación.

\section{Conclusiones}

Tanto en la agenda pública como en la academia cobra cada vez más importancia la formación permanente, especialmente la que está dirigida a los trabajadores: somos conscientes de la fuerte innovación tecnológica en el tejido productivo y del alargamiento de la vida laboral, así como de la necesidad de mejora de las cualificaciones de la población activa del país como vía de crecimiento económico. Por ello, no es de extrañar que la educación de adultos se constituya como una pieza clave de la política comunitaria de empleo, y seguramente lo será más en tiempos de crisis.

Para analizar la formación permanente contamos desde el año 2008 con una nueva fuente de información, la Encuesta sobre la Participación de la Población Adulta en las Actividades de Aprendizaje -EADA 2007. Al ser un instrumento diseñado ad hoc para el análisis de este fenómeno, es posible plantearse preguntas de investigación que hasta ahora se contestaban con una mayor dosis de inferencia, en parte por el escaso tamaño de las muestras y en parte por tener que suponer algunas características que no se hacían explícitas en los datos. Además, la EADA es un instrumento acordado por todos los países de la Unión Europea y con periodicidad quinquenal, lo que permitirá un nivel de comparación geográfico y temporal muy notable.

A partir del potencial de esta nueva base de datos, en el presente artículo se ha elaborado una tipología de formación poco utilizada en nuestro entorno. Así, a través del análisis de la educación no formal se ha estudiado el conjunto de la formación laboral, que va más allá de las actividades de formación de las empresas al integrar también las estrategias formativas de los individuos. En otras palabras, se han podido diferenciar las estrategias empresariales de las estrategias personales de aquellos trabajadores que se financian su propia formación. Además, gracias a la metodología basada en regresiones logísticas de eventos repetidos se ha podido apreciar si en cada tipo de formación la participación obedece más a la decisión personal o bien prevalecen factores estructurales relativos al tipo de puesto 0 al tipo de empresa en el que se desarrolla la actividad laboral.

Si nos referimos a los resultados obtenidos, en la formación financiada por la empresa a través de cursos y programas no se han apreciado diferencias significativas entre varones y mujeres. Eso sí, se destina la formación a los trabajadores más jóvenes 
mientras que los de mayor edad tienen muy pocas probabilidades de ser formados. También está dirigida a los trabajadores que poseen un mayor capital humano inicial: los universitarios. Parece que en conjunto las empresas forman a los más formados y a los que, se supone, pueden aprovechar mejor la inversión realizada por la empresa. En cuanto a las características de los puestos donde se recibe mayor formación, ésta es más intensa en las categorías ocupacionales que requieren altas cualificaciones. Y, en conjunto, se forma a los trabajadores con lazos más firmes con la organización; a aquellos con contrato fijo, de mayor antigüedad y en puestos a jornada completa. En cuanto a las características de las empresas destacan las de mayor tamaño y pertenecientes al sector financiero. Estos resultados indican una cierta polarización de los itinerarios formativos de los asalariados, así como una alta probabilidad de exclusión de la formación cuando a la baja cualificación del trabajador se une la edad. Asimismo, la polarización también se produce entre las empresas, con una desventaja para las más pequeñas y medianas que no pueden disponer de personal cualificado en la misma medida que las grandes.

En la adquisición de formación en el puesto de trabajo las características de la organización son aún más relevantes. Este aprendizaje está fuertemente asociado a la gran empresa. Las características del puesto resultan también factores explicativos, pues son los trabajadores varones con estudios de Formación Profesional de nivel superior y en puestos de nivel intermedio en la jerarquía ocupacional los que más reciben este tipo de formación; un fenómeno en el que seguramente tenga algo que decir la especialización funcional por género.

Se observan diferencias significativas según quién financie la formación. Las mujeres son las que tienen mayores probabilidades de realizar esta inversión por su cuenta y también los empleados más jóvenes. Asimismo cuanto mayor es el nivel educativo mayor es la iniciativa de acumular capital humano no financiado por la empresa. Se trata de empleados con vínculos débiles con la organización que posiblemente deseen estabilizarse y mejorar sus perspectivas de empleo y promoción realizando este tipo de inversiones formativas.

Por último, la formación no financiada por la empresa y realizada por motivos no relacionados con el trabajo está protagonizada en gran medida por trabajadores que compatibilizan trabajo y estudios; una situación que no se observa en los otros tipos de formación. Nos encontramos, pues, ante jóvenes inmersos todavía en la educación formal y que realizan alguna actividad laboral. En la misma línea de estos resultados apuntan las características de las empresas donde trabajan: empresas de pequeños tamaños y pertenecientes sobre todo al sector de la hostelería.

Además de las diferentes estrategias y sus tipologías, un objetivo implícito a la mayor parte de la investigación sobre formación de trabajadores y que no podemos eludir en estas páginas se refiere al efecto que la formación permanente tiene sobre el aumento o disminución de las oportunidades laborales entre los trabajadores. Se trata de ver si todos estos tipos de formación benefician especialmente a aquellos que parten de 
mejores posiciones en cuanto a capital humano, aumentando así las desigualdades de partida en el mercado de trabajo. Nuestros resultados son congruentes con la literatura especializada y nos señalan una realidad muy arraigada: la formación permanente a lo largo de la vida no iguala las diferencias de formación inicial sino que las intensifica. Las empresas destinan la formación a los trabajadores que poseen un mayor nivel educativo y estos asimismo son los que más invierten en formación como un mecanismo de obtención de mejoras laborales.

En este sentido, y teniendo en mente la necesidad de favorecer la formación en el conjunto de la fuerza laboral, el análisis de los que no se forman nos lleva a dos consideraciones: por una parte, una mayoría de los que expresamente manifiestan que no desean mayor formación esgrimen razones asociadas a la falta de tiempo, a las dificultades de gestionar los periodos formativos con los laborales o los domésticos. Por otra, aunque sean minoría, demasiados trabajadores se sienten ajenos a la formación o manifiestan la idea de que no la necesitan. En otras palabras, se perfilan dos líneas de acción pública: la necesidad de facilitar la formación a través de una mejor gestión de los tiempos de trabajo; y la sensibilización de buena parte de la población acerca de la importancia de la formación permanente.

No parece arriesgado suponer que con la crisis aumentará la demanda social para que los poderes públicos apuesten por la formación, no en vano es una de las más importantes políticas activas de empleo. En este contexto, parece necesario un esfuerzo por parte de todos (instituciones públicas y particulares) para que se entienda la formación como un importante capital productivo que además debe ir aumentando a lo largo de la vida. El problema se agudiza ante el hecho de que los asalariados que no quieren formarse son mayoritariamente los menos formados, en un país en el que casi un tercio de sus jóvenes no reciben formación reglada de tipo profesional, es decir no siguen ningún tipo de estudios después de los de secundaria obligatoria. A este respecto, sin erradicar el problema del fracaso escolar en España, parece muy difícil superar las desigualdades que la formación posterior genera entre los trabajadores.

\section{Referencias Bibliográficas}

Alba Ramírez, A. y M. Tugores Ques 2000. "Un estudio microeconómico sobre los determinantes y efectos de la formación en España." Pp. 341-420 en Formación y empleo: programa de economía familiar, coordinado por Felipe Sáez Fernández. Madrid: Fundación Argentaria.

Albert, C., C. García Serrano y V. Herranz. 2005. "Los determinantes de la Formación en la Empresa y sus Rendimientos." Comunicación presentada en las VI Jornadas de Economía Laboral. Julio, Alicante.

Altonji, J. G. y J. R. Spletzer. 1991. "Worker Characteristics, Job Characteristics, and the Receipt of on-the job Training." Industrial y Labor Relations Review 45:58-79. 
Aris Redó, N. y M. A. Comas. 2011. "La formación permanente en el contexto del Espacio Europeo de la Formación Permanente." Revista de Universidad y Sociedad del Conocimiento (RUSC) 2:5-13. Consulta 3 de julio de 2012 (http://rusc.uoc.edu/ojs/index.php/rusc/article/view/v8n2-aris-comas/v8n2).

Arrow, K. J. 1991. "La educación superior como filtro." Economía y Sociedad 8:143-161.

Baltagi, B. 1995. Econometric Analysis of Panel Data. Nueva York: John Wiley \& Son.

Barba Aragón, M. I., A. Aragón Sánchez y R. Sanz Valle. 2000. "Condicionantes de la formación en las PYMES industriales." Economía Industrial 334:35-43.

Barret, A. y P. J. O'Connell. 2001. "Does training generally work? The returns to in-company training." Industrial and Labor Relations Review 54:647-662.

Becker, G. 1983. El Capital Humano. 2. ${ }^{a}$ ed. Madrid: Alianza Editorial.

Booth, A. L. 1991. "Job-Related Formal Training: who receives it and what is it worth?" Oxford Bulletin of Economics and Statistics 53:281-293.

Booth, A. L. 1993. "Private Sector Training and Graduate Earnings." The Review of Economics and Statistics 75:164-170.

Caparrós Ruiz, A., M. L. Navarro Gómez y M. F. Rueda Narváez. 2004. "Efectos de la temporalidad sobre la formación recibida durante el empleo." Cuadernos de Economía 27:51-74.

Caparrós Ruiz, A., M. L. Navarro Gómez y M. F. Rueda Narváez. 2009. "Análisis de la incidencia y duración de la formación laboral financiada por empresas y trabajadores." Cuadernos de Economía 32:264-278.

Caparrós Ruiz, A., M. L. Navarro Gómez y M. F. Rueda Narváez. 2010. "Rentabilidad salarial de la formación laboral: un análisis con datos de panel." Estudios de Economía Aplicada 28:1-20.

Cedefop (European Centre for the Development of Vocational Training). 2010. "Employer-provided vocational training in Europe." Research Paper 2. Luxembourg: Publications Office of the European Union. Consulta 10 de diciembre de 2011 (http://www.cedefop.europa.eu/EN/Files/5502_en.pdf).

Cedefop (European Centre for the Development of Vocational Training). 2011. "The impact of vocational education and training on company performance." Research Paper 19. Luxembourg: Publications Office of the European Union. Consulta 10 de diciembre de 2011 (http://www.cedefop.europa.eul EN/Files/5519_en.pdf).

Colom Cañellas, A. J. 2005. "Continuidad y complementariedad entre la educación formal y no formal." Revista de Educación 338:9-22.

Doeringer, P. B. y M. J. Piore. 1985. Mercados internos de trabajo y análisis laboral ed. Madrid: Ministerio de Trabajo.

Dustmann, C. y U. Schönberg. 2009. "Training and Union Wages." The Review of Economics and Statistics 9:363-376.

Escobar, M., J. Rivière y L. Glick. 2010. "La distribución social de la formación permanente laboral en España". Revista Española de Sociología (RES) 14:43-65.

European Society of Association Education. 2005. What is Lifelong Learning? The view from the European Commission, European Society of Association Education. Consulta 30 septiembre 2010 (http://www.esae.org/articles/2007_08_005.pdf). 
Frazis, H. J., D. E. Herz y M. W. Horrigan. 1995. "Employer-provided training: results from a new survey." Monthly Labor Review 118:3-17.

Frazis, H. J., M. Gittleman y M. Joyce. 2000. "Correlates of training: an analysis using both employer and employee characteristics." Industrial and Labor Relations Review 53:443462.

Fundación Tripartita para la Formación en el Empleo 2010. Memoria 2010. Consulta 3 de julio de 2012 (http://www.fundaciontripartita.org/index.asp?MP=5\&MS=93\&MN=2).

García Espejo, I. 1999. "Formación en el trabajo y movilidad laboral." Papers 59:195-219.

Green, F. 1993. "The Determinants of Training of Male and Female Employees in Britain." Oxford Bulletin of Economics and Statistics 55:103-122.

Greenhalgh, C. y G. Mavrotas 1994. "The Role of Career Aspirations and Financial Constraints in Individual Acces to Vocational Training." Oxford Economic Papers 46:579-601.

Huerta Arribas, E. 2003. Los desafíos de la competitividad. La innovación organizativa y tecnológica de la empresa española. Bilbao: Fundación BBVA.

Ibáñez Pascual, M. 1999. Género y familia en la inserción laboral. Oviedo: Universidad de Oviedo.

Instituto Nacional de Estadística. 2008a. Encuesta sobre la Participación de la Población Adulta en las Actividades de Aprendizaje (EADA 2007). Notas de Prensa. Madrid: INE. Consulta 15 enero de 2012 (http://www.ine.es/prensa/np496.pdf).

Instituto Nacional de Estadística. 2008b. Encuesta sobre la Participación de la Población Adulta en las Actividades de Aprendizaje (EADA 2007). Informe metodológico. Madrid: INE. Consulta 15 enero de 2012 (http://www.ine.es/metodologia/t13/t133045907.pdf).

Jonker, N. y A. de Grip 1999. "Do Employees with Flexible Contracts Receive less Training?” Documento de trabajo. Universidad de Maastricht. Consulta 28 diciembre 2011. (http://arno.unimaas.nl/ show.cgi?fid=731).

Kirkpatrick, D. L. 1999. Evaluación de acciones formativas. Los cuatro niveles. Barcelona: EPISE.

Knoke, D. y A. L. Kalleberg. 1994. "Job Training in U.S. Organizations." American Sociological Review 59:537-546.

Lynch, L. M. y S. E. Black. 1998. "Beyond the incidence of employer-provide training." Industrial and Labor Relations Review 52:64-81.

Lynch, L. M. 1992. "Private-Sector Training and the Earnings of Young Workers." American Economic Review 82:299-312.

Martín Artiles, A. y A. Lope. 1999. “¿Sirve la formación para tener empleo?." Papers 58:39-73.

Maruani, M. 1993. "La cualificación, una construcción social sexuada." Revista de Economía y Sociología del Trabajo 21-22:41-50.

Mato, F. J. 2002. La formación para el empleo: una evaluación cuasi-experimental. Madrid: Civitas.

Mato, F. J. 2010. "La formación continua en España desde una perspectiva comparada: balance y propuestas de mejora." Papeles de Economía Española 124:266-280. 
Mayorga, M. y E. Muñoz 2000. "La técnica de datos de panel. Una guía para su uso e interpretación." Documento de trabajo (DIE-NT-05-2000), Banco Central de Costa Rica, San José. (http://www.seti. chubut.gov.ar/Publico/PDF/Mayorga $\% 20 \mathrm{y} \% 20 \mathrm{Mu} \%$ C3\%B10z $\% 20$ Tecnica $\% 20$ de $\% 20$ datos $\% 20$ de\%20panel.pdf).

Ministerio de Educación de España. 2010. El aprendizaje permanente en España. Consulta 4 de julio de 2012. (http://www.educacion.gob.es/dctm/ministerio/horizontales/prensa/documentos/2011/11/ el-aprendizaje-permanente-en-espan?documentld=0901e72b81071ba3).

Peraita, C. 2000. "Características de la formación en la empresa española." Papeles de Economía Española 86:295-307.

Pineda, P. H. 2000. "Evaluación del impacto de la formación en las organizaciones." Educar 27:119-133.

Planas, J. y J. Rifá 2001. "The Continuing Training in Spain: Institucional Logic and Individual Usage." Les Cahiers du Lasmas 1:45-61.

Sáez, F. 2002. "Capital humano, innovación y empleo. Su adecuación." Economía industrial 348:67-75.

Spence, M. 1991. "Indicadores del mercado de trabajo." Educación y Sociedad 8:123-141.

Thurow, L. 1975. Generating Inequality. Nueva York: Basic Books.

Tugores, M. y A. Alba 2002. "Estudio microeconómico sobre la formación continua en España. 19922002." Economía Industrial 348:77-94.

Unión Europea 1996. Síntesis de la legislación de la UE. Año Europeo de la Educación y de la Formación Permanentes. Consulta 20 enero 2012. (http://europa.eu/legislation_summaries/education_training_youth/lifelong_learning/c11024_es.htm).

Isabel García Espejo es licenciada en Sociología por la Universidad Complutense de Madrid y Doctora en Sociología por la Universidad de Oviedo. Es profesora titular de Sociología en la Facultad de Economía y Empresa de la Universidad de Oviedo. Imparte docencia en Técnicas de Investigación Social desde 1988, además de en otras materias del campo de la Sociología. $\mathrm{Ha}$ realizado investigaciones y publicado diversos trabajos sobre inserción laboral de jóvenes, formación y mercado de trabajo, movilidad laboral, formación en la empresa, desigualdades sociales, técnicas de investigación cuantitativas, análisis de datos longitudinales y sociología de la alimentación.

Marta Ibañez Pascual es Titular de Sociología en la Universidad de Oviedo. Su investigación enlaza los campos de la sociología del trabajo y la inserción laboral, las diferencias por género y la sociología de la familia. Página web: http://www.unioviedo.es/mibanez/.

\section{RECIBIDO: $12 / 03 / 2012$}

ACEPTADO: 16/10/2012

Publicado on-line: 12/04/2013 